\title{
Search for Low-Mass Axion Dark Matter with ABRACADABRA-10 cm
}

\author{
Chiara P. Salemi, ${ }^{1,{ }^{*}, \dagger}$ Joshua W. Foster, ${ }^{2,3,4, *,+}$ Jonathan L. Ouellet, ${ }^{1,{ }^{*},}$ Andrew Gavin, ${ }^{5}$ Kaliroë M. W. Pappas, ${ }^{1}$ \\ Sabrina Cheng, ${ }^{1}$ Kate A. Richardson, ${ }^{5}$ Reyco Henning, ${ }^{5,6}$ Yonatan Kahn, ${ }^{7,8}$ Rachel Nguyen, ${ }^{7,8}$ Nicholas L. Rodd, \\ Benjamin R. Safdi, ${ }^{2,3,4}$ and Lindley Winslow ${ }^{1, \|}$ \\ ${ }^{1}$ Laboratory of Nuclear Science, Massachusetts Institute of Technology, Cambridge, Massachusetts 02139, USA \\ ${ }^{2}$ Leinweber Center for Theoretical Physics, Department of Physics, University of Michigan, Ann Arbor, Michigan 48109, USA \\ ${ }^{3}$ Berkeley Center for Theoretical Physics, University of California, Berkeley, California 94720, USA \\ ${ }^{4}$ Theoretical Physics Group, Lawrence Berkeley National Laboratory, Berkeley, California 94720, USA \\ ${ }^{5}$ Department of Physics and Astronomy, University of North Carolina, Chapel Hill, Chapel Hill, North Carolina 27599, USA \\ ${ }^{6}$ Triangle Universities Nuclear Laboratory, Durham, North Carolina 27710, USA \\ ${ }^{7}$ Department of Physics, University of Illinois at Urbana-Champaign, Urbana, Illinois 61801, USA \\ ${ }^{8}$ Illinois Center for Advanced Studies of the Universe, University of Illinois at Urbana-Champaign, Urbana, Illinois 61801, USA
}

(Received 31 March 2021; accepted 16 July 2021; published 17 August 2021)

\begin{abstract}
Two of the most pressing questions in physics are the microscopic nature of the dark matter that comprises $84 \%$ of the mass in the Universe and the absence of a neutron electric dipole moment. These questions would be resolved by the existence of a hypothetical particle known as the quantum chromodynamics (QCD) axion. In this work, we probe the hypothesis that axions constitute dark matter, using the ABRACADABRA-10 $\mathrm{cm}$ experiment in a broadband configuration, with world-leading sensitivity. We find no significant evidence for axions, and we present $95 \%$ upper limits on the axionphoton coupling down to the world-leading level $g_{\text {ary }}<3.2 \times 10^{-11} \mathrm{GeV}^{-1}$, representing one of the most sensitive searches for axions in the $0.41-8.27 \mathrm{neV}$ mass range. Our work paves a direct path for future experiments capable of confirming or excluding the hypothesis that dark matter is a QCD axion in the mass range motivated by string theory and grand unified theories.
\end{abstract}

DOI: 10.1103/PhysRevLett.127.081801

The axion is a well-motivated candidate to explain the particle nature of dark matter (DM) [1-3]. This pseudoscalar particle is naturally realized as a pseudo-Goldstone boson of the Peccei-Quinn symmetry, which is broken at a high scale $f_{a}$; the axion would be exactly massless but for its low-energy interactions with quantum chromodynamics (QCD) [4-7]. The axion mass is tied to the scale $f_{a}$ by $m_{a} \approx 5.7\left(10^{15} \mathrm{GeV} / f_{a}\right) \mathrm{neV}[8]$. The range of scales $f_{a} \approx$ $10^{15}-10^{16} \mathrm{GeV}$ is particularly compelling because of connections to string theory [9] and grand unification [10,11], and in the corresponding mass range of $m_{a} \sim 1-10 \mathrm{neV}$ the axion may naturally explain the observed DM abundance $[11,12]$. In this Letter, we provide the most sensitive probe of axion dark matter (ADM) in this mass range to date.

ADM that couples to photons modifies Ampère's law such that in current-free regions

$$
\nabla \times \mathbf{B}=\frac{\partial \mathbf{E}}{\partial t}-g_{a \gamma \gamma}\left(\mathbf{E} \times \nabla a-\frac{\partial a}{\partial t} \mathbf{B}\right)
$$

Published by the American Physical Society under the terms of the Creative Commons Attribution 4.0 International license. Further distribution of this work must maintain attribution to the author(s) and the published article's title, journal citation, and DOI. Funded by SCOAP. with $\mathbf{E}$ and $\mathbf{B}$ the electric and magnetic fields, respectively, $a(\mathbf{x}, t)$ the ADM field, and $g_{a y \gamma}$ the axion-electromagnetic coupling constant. In the presence of a static external magnetic field, ADM behaves like an effective current

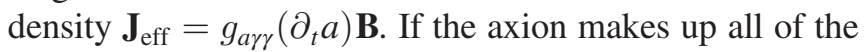
observed DM, then, to leading order in the DM velocity, $\partial_{t} a=\sqrt{2 \rho_{\mathrm{DM}}} \cos \left(m_{a} t\right)$, with $\rho_{\mathrm{DM}} \approx 0.4 \mathrm{GeV} / \mathrm{cm}^{3}$ the local DM density [13]. It was pointed out in Refs. $[14,15]$ that the effective current induces an oscillating secondary magnetic field which may be detectable in the laboratory without the aid of a resonant cavity for sufficiently small $m_{a}$. The oscillation frequency is given by $f=m_{a} /(2 \pi)$, with bandwidth $\delta f / f \approx 10^{-6}$ arising from the finite axion velocity dispersion [16]. In this work, we leverage this theoretical principle to search for axions in the laboratory.

The most common detection strategy for ADM is through the electromagnetic coupling $g_{a \gamma \gamma}$, which for the QCD axion is directly proportional to the mass $m_{a}$. Until recently, experiments have focused on searching for axions in the mass range $1 \lesssim m_{a} \lesssim 40 \mu \mathrm{eV}$, which is well suited to microwave cavity searches [17-21]. In the low-mass regime targeted in this work, the Compton wavelength of the axion $\lambda_{C} \sim \mathrm{km}$ is much larger than the experimental apparatus, and so the sensitivity of the experiment improves 
with volume as $V^{5 / 6}$, roughly independent of $m_{a}$ until the size of the experiment approaches $\lambda_{C}$ [15]. This scaling is important, because the expected coupling $g_{a \gamma \gamma}$ is smaller at lower masses, requiring ever-more-sensitive experiments to achieve a detection. ABRACADABRA is an experimental program designed to detect axions at the grand unification scale using a strong toroidal magnetic field [15]. ABRACADABRA is part of a suite of ADM experiments which together aim to probe the full QCD axion parameter space [19-27]. The experiment we report on here, ABRACADABRA-10 cm, is a prototype for a larger ADM detector that would be sensitive to the QCD axion. This Letter presents data collected in 2020 that is up to an order of magnitude more sensitive than our previous results [28] and places strong limits on ADM in the $0.41-8.27 \mathrm{neV}$ range of axion masses.

ABRACADABRA-10 cm detector.-The ABRACADABRA$10 \mathrm{~cm}$ detector is built around a 12-cm-diameter, $12-\mathrm{cm}-$ tall, $1 \mathrm{~T}$ toroidal magnet fabricated by Superconducting Systems Inc. [29]. The axion interactions with the toroidal magnetic field $\mathbf{B}_{0}$ drive the effective current, $\mathbf{J}_{\text {eff }}$, which oscillates parallel to $\mathbf{B}_{0}$ and sources a real oscillating magnetic field through the toroid's center. The oscillating magnetic flux is read out with a two-stage dc superconducting quantum interference device (SQUID) via a superconducting pickup in the central bore. Unlike other axion detector designs, this novel geometry situates the readout pickup in a nominally field-free region unless axions are present [15]. The detector can be calibrated by injecting fake axion signals (i.e., ac currents) through a wire calibration loop that runs through the body of the magnet. The detector, illustrated schematically in Fig. 1, is located on MIT's campus in Cambridge, Massachusetts.

In 2019, we performed several detector upgrades from the run 1 configuration in order to improve our sensitivity [28,31]. In this Letter, we report the results of the subsequent data campaign (run 3), collected after the detector upgrade. Run 3 data consist of $\sim 430 \mathrm{~h}$ of data collected from June 5 to June 29, 2020.

Before the upgrades were complete, we took additional, uncalibrated data (run 2), which is not presented here. A subset of that data was instead used to develop our data analysis procedure in order to run a blind analysis on the run 3 data, as described in detail below.

The total expected axion power $A$ coupled into our readout pickup is related to the axion-induced flux $\Phi_{a}$ as

$$
A \equiv\left\langle\left|\Phi_{a}\right|^{2}\right\rangle=g_{a \gamma \gamma}^{2} \rho_{\mathrm{DM}} \mathcal{G}^{2} V^{2} B_{\max }^{2},
$$

where $\mathcal{G}$ is a geometric coupling, $V$ is the magnetic field volume, $B_{\max }$ is the maximum value of $\left|\mathbf{B}_{0}\right|$, and the angle brackets denote the time average [15,32]. Run 1 utilized a 4.02-cm-diameter pickup loop made from a 1-mm-diameter wire, giving $\mathcal{G} \approx 0.027$. In 2019 , we replaced this readout with a 10-cm-tall, 5.1-cm-diameter superconducting
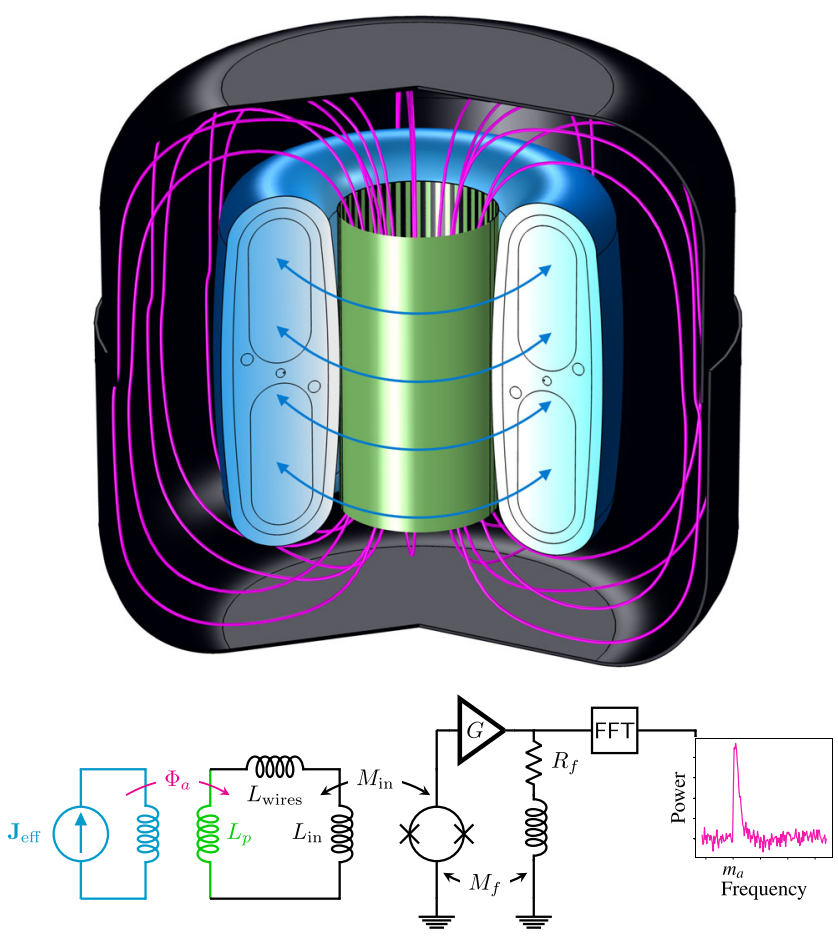

FIG. 1. Top: schematic of ABRACADABRA-10 $\mathrm{cm}$ showing the effective axion-induced current (blue), sourced by the toroidal magnetic field, generating a magnetic flux (magenta) through the pickup cylinder (green) in the toroid bore. Bottom: simplified schematic of the ABRACADABRA-10 $\mathrm{cm}$ readout (full circuit diagram in Supplemental Fig. S1 [30]). The pickup cylinder $L_{p}$ is inductively coupled to the axion effective current $\mathbf{J}_{\text {eff }}$. The power spectrum of the induced current is read out through a dc SQUID inductively coupled to the circuit through $L_{\mathrm{in}}$. An axion signal would appear as excess power above the noise floor at a frequency corresponding to the axion mass.

cylinder pickup centered in the toroid bore. This consisted of a $150-\mu \mathrm{m}$-thick $\mathrm{Nb}$ sheet wrapped around a polytetrafluoroethylene cylinder. This design yields a stronger geometric coupling to $\mathbf{J}_{\text {eff }}$ of $\mathcal{G} \approx 0.031$ and decreases the inductance of the pickup [15]. We compute $\mathcal{G}$ using electromagnetic simulations in the COMSOL MULTIPHYSICS package $[31,33]$.

To amplify our signal, $\Phi_{a}$ is coupled into the readout SQUID through the pickup circuit (see Fig. 1) yielding a transformer gain $M_{\text {in }} / L_{T}$, where $M_{\text {in }}$ is the input coupling to the SQUID and $L_{T} \equiv L_{p}+L_{\text {in }}+L_{\text {wires }}$ is the total inductance of the pickup circuit, with $L_{p}$ the pickup cylinder inductance, $L_{\mathrm{in}}$ the input inductance of the SQUID package, and $L_{\text {wires }}$ the parasitic inductance, dominated by the twisted pair wiring. The SQUID, manufactured by Magnicon [34], is read out using Magnicon's XXF-1 SQUID electronics operating in closed feedback loop mode. The run 1 sensitivity was limited by parasitic inductance in the NbTi wiring of this circuit that placed a lower limit on $L_{T} \gtrsim 1.6 \mu \mathrm{H}$. During the upgrade, we replaced this wiring, moving the SQUIDs closer to the 
detector to reduce the wire length. Based on calibration data, we found that the total impedance in the circuit is $\sim 800 \mathrm{nH}$. Finally, the SQUID was operated at a higher flux-to-voltage gain setting of $4.3 \mathrm{~V} / \Phi_{0}$ in run 3 , compared to the previous run 1 , which we ran at $1.29 \mathrm{~V} / \Phi_{0}$ due to higher levels of environmental noise. This change does not directly improve the signal gain but does reduce system noise. We also improved our noise floor by reducing the operating temperature of the SQUID package from $~ 870$ to $\sim 450 \mathrm{mK}$. All together, the upgrade campaign increased the expected power coupled into our readout and reduced the total system noise.

The improved sensitivity of the upgraded readout circuit also amplified the low-frequency vibrational backgrounds seen in run 1, which caused the SQUID amplifier to rail when the magnet was on. In order to correct this, we implemented an active feedback stabilization system to reduce the low-frequency noise, which is discussed further in Supplemental Material [30].

As in run 1, the magnet and pickup were placed inside a superconducting tin-copper shield and hung from a passive vibration isolation system, consisting of a string pendulum and spring, within an Oxford Instruments Triton 400 dilution refrigerator [31]. The magnet and pickup were operated at $\lesssim 1 \mathrm{~K}$, and the SQUIDs were at $\sim 400 \mathrm{mK}$, which kept the readout circuit superconducting over the course of the run and kept thermal noise subdominant to SQUID flux noise. Following the procedure of run 1, the output of the SQUID was run into an 8-bit AlazarTech AT9870 digitizer via a $70 \mathrm{kHz}-5 \mathrm{MHz}$ bandpass filter. The digitizer was locked to a Stanford Research Systems FS725 rubidium frequency standard in order to maintain clock accuracy over the coherence time of the axion signal, $\sim 1 \mathrm{~s}$ for signals at $1 \mathrm{MHz}$, throughout the data and calibration runs.

We performed in situ magnet-on and magnet-off calibrations in the data-taking configuration by attaching a harmonic signal generator to the calibration circuit and scanning across frequencies and amplitudes. The calibration signal was attenuated and fed into the calibration loop, mimicking the axion effective current signal $\mathbf{J}_{\text {eff }}$ up to geometric factors. The geometry is modeled in COMSOL MULTIPHYSICS [33], from which we extract the coupling between both the calibration loop and axion effective current signal to the pickup cylinder. By combining the results of the calibration scans and geometric modeling, we can determine the effective gain $\partial V_{\text {out }} / \partial \Phi_{a}$ of the SQUID amplifier output voltage as a function of flux on the pickup cylinder (see Fig. 2). This procedure is analogous to that used in run 1 [31].

The gain measured by the calibrations for run 3 differs from the calculated gain by a factor of $\sim 1.8$. By individually calibrating various components of the end-to-end circuit, we determined that this is likely due to a misestimation of the calculated total inductance of the pickup

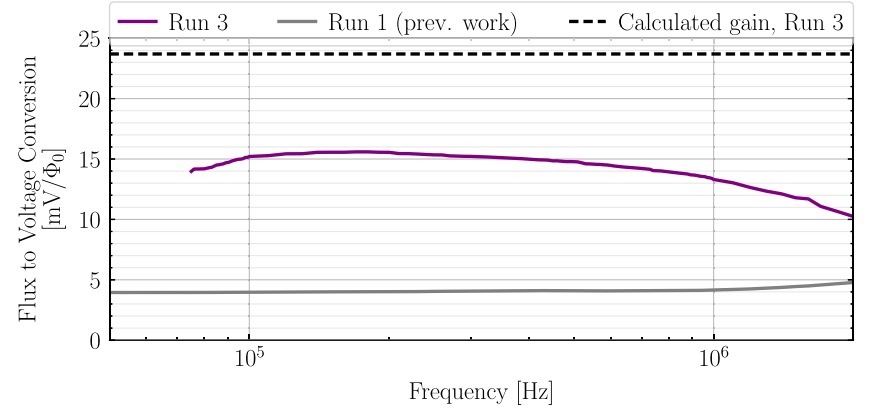

FIG. 2. The gain shown here is defined as the change in amplifier output voltage over a corresponding change in input flux amplitude on the pickup cylinder $\left(\partial V_{\text {out }} / \partial \Phi_{a}\right)$. Both transfer functions roll off at high frequencies due to the amplifier bandwidth, which we estimate to have a cutoff frequency of approximately $1 \mathrm{MHz}$. We believe the difference in calculated and measured gain is due to inconsistency in the total inductance of the pickup circuit.

circuit. The calibrated SQUID noise floors, which set the lower limits of our sensitivity, are shown in Supplemental Fig. S2 [30].

Data collection.-The axion search data were collected using an identical procedure as in run 1 [31]. The SQUID amplifier output voltage was sampled at a frequency of $10 \mathrm{MS} / \mathrm{s}$, with a $\pm 40 \mathrm{mV}$ voltage window. The data were stored as a series of power spectral densities (PSDs), which were computed on the fly: $\overline{\mathcal{F}}_{10 \mathrm{M}}$ with a Nyquist frequency of $5 \mathrm{MHz}$ and frequency resolution of $\Delta f=100 \mathrm{mHz}$, $\overline{\mathcal{F}}_{1 \mathrm{M}}$ with a Nyquist frequency of $500 \mathrm{kHz}$ and frequency resolution of $\Delta f=10 \mathrm{mHz}$, and a continuous data stream sampled at $100 \mathrm{kHz}$ that can be analyzed offline. $\overline{\mathcal{F}}_{10 \mathrm{M}}$ $\left(\overline{\mathcal{F}}_{1 \mathrm{M}}\right)$ is averaged over $800 \mathrm{~s}(1600 \mathrm{~s})$ before being written to disk. In this work, we used the $\overline{\mathcal{F}}_{10 \mathrm{M}}$ to search the frequency range from $500 \mathrm{kHz}$ to $2 \mathrm{MHz}$ and the $\overline{\mathcal{F}}_{1 \mathrm{M}}$ spectra to search from 50 to $500 \mathrm{kHz}$.

Data analysis and results.-An axion signal is expected to manifest as a narrow peak in the PSD data, as illustrated in Fig. 1. The width and overall shape of the signal are set by the local DM velocity distribution, which we take to be the standard halo model with a velocity dispersion of $v_{0}=$ $220 \mathrm{~km} / \mathrm{s}$ and a boost from the halo to the solar rest frame of $v_{\odot}=232 \mathrm{~km} / \mathrm{s}$ [35]. With the speed distribution and local DM density fixed, the two free signal parameters are the axion mass $m_{a}$, which determines the minimum frequency of the signal, and the coupling $g_{a \gamma \gamma}$, which determines its amplitude through Eq. (2). Our analysis procedure closely follows the approach used in the run 1 search [28,31] based on Ref. [32], which constrains the allowable values of $g_{a y \gamma}$ at each possible value of $m_{a}$.

The search is performed with a frequentist log-likelihood ratio test statistic (TS); exact expressions are provided in Supplemental Material [30] (see also [31]). Our broadband search procedure probes $\sim 11.1$ million mass points 
between 0.41 and $8.27 \mathrm{neV}(100 \mathrm{kHz}-2 \mathrm{MHz})$ in run 3. As we expect only one axion signal in our search (or at most a small number), the majority of the TS values are probing the distribution of the null hypothesis. Assuming only Gaussian noise, we expect this null distribution to be a onesided $\chi^{2}$ distribution [32], which was indeed the case in run 1 [28,31]. However, the increased sensitivity from the detector upgrades introduced non-Gaussian noise sources that required us to modify our run 1 analysis procedure. We developed and validated our new procedure on a randomly selected sample of $10 \%$ of run 2's 13.7 million mass points, after which we unblinded the run 3 data with the procedure fixed.

In run 1, we searched for an axion signal as a feature appearing above a flat white noise background. For each $m_{a}$, the search was performed in a narrow window around that mass with the background level allowed to vary independently in each window. For the run 2 and run 3 analyses, we allow the mean background level of the noise to vary linearly with frequency uniquely in each sliding window. We use sliding windows of relative width $\delta f / f \approx 5.5 \times 10^{-6}$, starting at $f=\left(1-10^{-6}\right) \times m_{a} /(2 \pi)$.

As in run 1, we use the magnet-off data to veto frequency ranges that also display statistically significant TS values when $\left|\mathbf{B}_{0}\right|=0$, and, thus, the axion power should vanish. However, we observed narrow single-bin "spikes" that appear to drift in frequency on the timescale of our data collection (see Supplemental Fig. S6 [30] for an example). If interpreted in isolation, these spikes sometimes correspond to statistically significant excesses. Nevertheless, they are inconsistent with axion signals and are most likely due to unknown environmental noise sources near the detector, persisting throughout runs 2 and 3; indeed, many of the peaks are distributed at multiples of $50 \mathrm{~Hz}$. To remove these artifacts, we leverage the fact that the PSDs are saved periodically to disk, yielding a time evolution of the environmental backgrounds; we veto single-bin spikes that move in frequency. We place a $1.0 \mathrm{~Hz}$ veto window around these single-bin spikes. These cuts remove $3.8 \%$ of the axion mass points from our search in the run 3 data. The magnet-off vetoing procedure removes an additional $0.07 \%$ of mass points.

After implementing the vetoes, we found the distribution of TS values in the $10 \%$ run 2 validation sample deviated from the expected $\chi^{2}$ distribution; for example, there were 27 mass points with $\mathrm{TS}>25$, whereas from the $\chi^{2}$ distribution we would have expected less than one. To account for the deviation in the TS distribution from the $\chi^{2}$ distribution in a data-driven fashion, we follow the prescription developed and implemented in Refs. [36-38] for searches for DM-induced lines in astrophysical gamma-ray datasets. At each mass point, we introduce and profile over a systematic nuisance parameter that would be degenerate with the signal parameter but for a prior that is determined by forcing the TS distribution to follow the $\chi^{2}$ distribution.
Specifically, we force the TS distribution to match the null hypothesis distribution at $4 \sigma$ local significance. This is described further in Supplemental Material [30].

After the nuisance parameter and vetoing procedures, we construct a likelihood as a function of $g_{a \gamma \gamma}$ at each mass point. The final distribution of TS values computed from the likelihoods is shown in Fig. 3; no TS values were found in excess of the $5 \sigma$ look-elsewhere effect-corrected discovery threshold. In the calibration of our analysis procedure, we found one signal candidate in the run 2 data at over $5 \sigma$ global statistical significance (see Supplemental Fig. S6 [30], where a corresponding feature can be seen in the magnet-off data), but that mass point is not significant in the run 3 analysis.

In the absence of an excess consistent with an ADM origin, we can determine $95 \%$ one-sided upper limits on $g_{a \gamma \gamma}$ as a function of the mass $m_{a}$. The average $95 \%$ upper limits from the run 3 analysis along with their $1 \sigma$ and $2 \sigma$ expectations under the null hypothesis are indicated in Fig. 4. In that figure, we compare our upper limits to those found from the ADM experiment SHAFT [26] along with results from the solar axion experiment CAST [39] and astrophysical x-ray searches (SSC) [40], both of which do not require the axion to comprise the DM. The fraction of vetoed mass points is illustrated in a sliding window in Supplemental Fig. S4 [30], which also shows the distribution of data fractions included in the analyses. In Supplemental Fig. S5 [30], we illustrate the magnitude of the systematic nuisance parameter $g_{a \gamma \gamma}^{\text {nuis }}$, while in

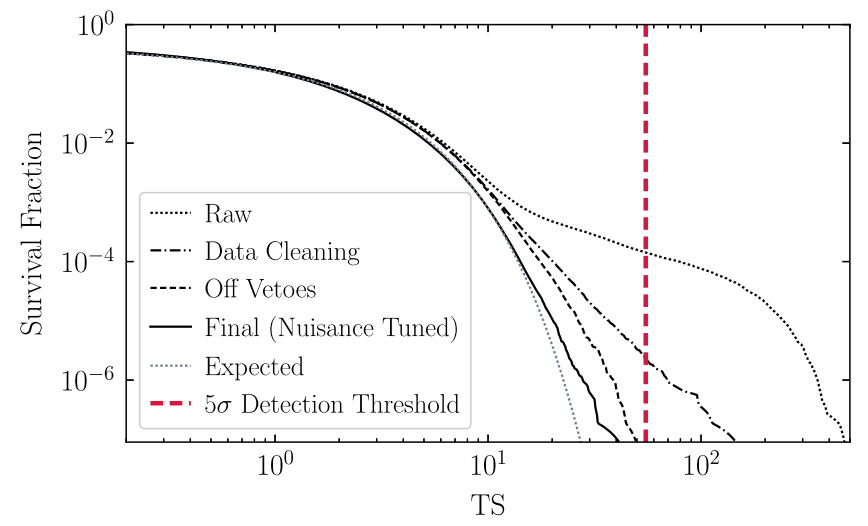

FIG. 3. The survival function of TS values from the likelihood analysis of the run 3 results. The $y$ axis indicates the fraction of mass points tested with a discovery TS at or above the value on the $x$ axis. Under the null hypothesis, the distribution should follow the survival function of the one-sided $\chi^{2}$ distribution with one degree of freedom ("expected," dotted gray line). This is indeed the case after data cleaning for, e.g., single-channel excesses in time slices, magnet-off vetoes, and the inclusion of a systematic nuisance parameter, which is tuned in a sliding window at $4 \sigma$ local significance to give the correct number of excesses at or above that significance, masking the signal of interest. No excesses are found beyond our indicated $5 \sigma$ lookelsewhere effect-corrected discovery threshold. 


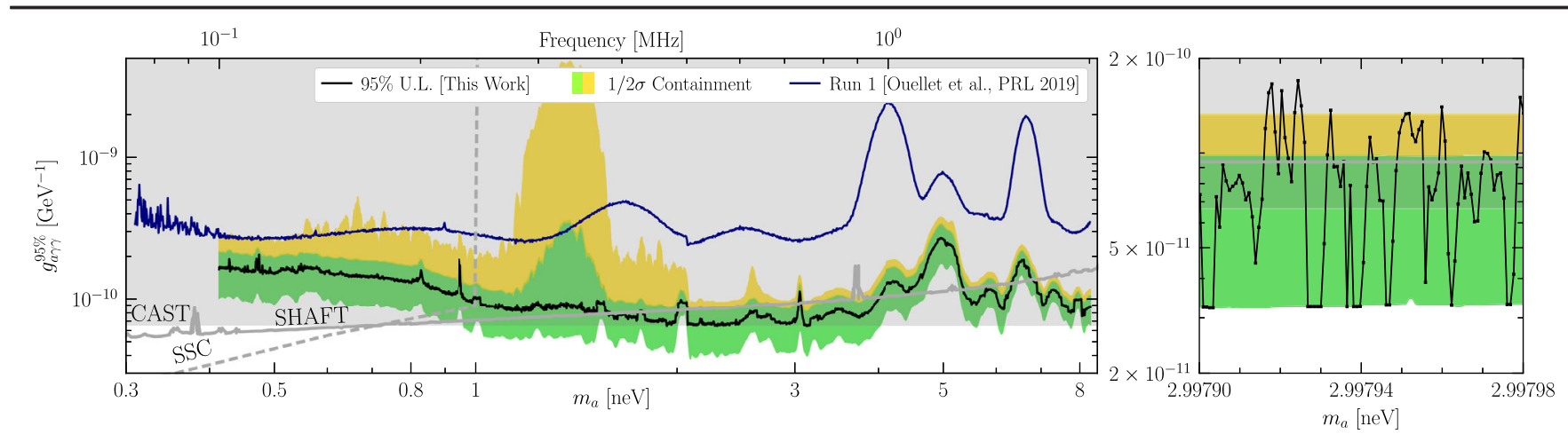

FIG. 4. Left: The one-sided 95\% upper limit (U.L.) on $g_{a \gamma \gamma}$ from this work excludes previously unexplored regions of ADM parameter space. The $1 \sigma$ and $2 \sigma$ containment regions are constructed by taking the appropriate percentiles of the distributions of the limits over narrow mass ranges; note that this means that $\sim 16 \%$ of the upper limits lie at the bottom of the green band. Around 11.1 million mass points are analyzed, so the plotted data are smoothed for clarity. Our limits surpass those from a number of indicated astrophysical and laboratory searches in this mass range (see the text for details). Right: the unsmoothed limit in a narrow mass range between 2.99790 and $2.99798 \mathrm{neV}$. This provides a detailed view of variations in the limit at each axion mass that arise from statistical fluctuations across the collected data that are not visible in the smoothed data shown in the left plot. This range also depicts the location where our

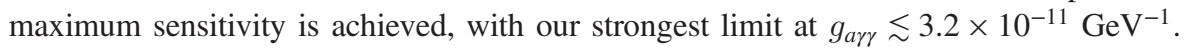

Supplemental Fig. S7 [30] we show what the limit would be without the nuisance parameter tuning. Supplemental Fig. S8 [30] shows that the 95\% upper limit and discovery TS behave as expected when synthetic axion signals are injected into the real data.

Discussion.-In this Letter, we present the results from ABRACADABRA-10 cm's second physics campaign, searching for ADM in the mass range 0.41-8.27 neV. We find no evidence for ADM and constrain the axionphoton coupling down to the world-leading level $g_{\text {ary }} \lesssim$ $3.2 \times 10^{-11} \mathrm{GeV}^{-1}$ at $95 \%$ confidence. Our work motivates key elements of the design of future larger-scale experiments. These include the mitigation of stray fields from the magnet and vibrations induced by a modern pulsetube-based cryogenic system, which limits our current lowfrequency reach. The ABRACADABRA-10 cm results presented in this Letter demonstrate the power of mature simulations for optimizing the design of the detector and for modeling the calibration response. An advanced and novel analysis framework was used to identify noise sources and account for systematic uncertainties in a datadriven fashion.

Our work identifies three areas that can be addressed in the next physics campaign: (i) moderate improvements (up to a factor of $\sim 0.4$ in $g_{\text {ary }}$ ) could be achieved by further reducing the wire and SQUID inductances, (ii) better shielding from environmental noise could increase the sensitivity to $g_{\text {ary }}$ by an order of magnitude at low frequencies, so long as (iii) the fringe fields are reduced or better vibrationally isolated (see Supplemental Fig. S2 [30]). To significantly increase the sensitivity of the experiment, larger magnets with higher fields are needed, since the sensitivity to $g_{a \gamma \gamma}$ scales with the detector volume $V$ and field $B_{0}$ as $g_{a \gamma \gamma}^{-1} \sim B_{0} V^{5 / 6}[15]$. The addition of a resonant readout circuit could enhance the reach in $g_{a \gamma \gamma}$ by an additional $\sim 2$ orders of magnitude depending on the scanning strategy, with a high-frequency readout permitting sensitivity to masses up to $800 \mathrm{neV}$ [15,41]. ABRACADABRA is merging with the DMRadio program to realize a series of experiments that chart a path toward discovering the QCD axion in the parameter space corresponding to new physics at the grand unification scale [42-46].

We thank Kent Irwin and our DMRadio colleagues for useful discussions and look forward to the next-generation experiment. We thank those that took part in run 1 of ABRACADABRA-10 $\mathrm{cm}$ including Zachary Bogorad, Janet Conrad, Joseph Formaggio, Joe Minervini, Alexey Radovinsky, Jesse Thaler, and Daniel Winklehner. We thank Christopher Dessert for useful analysis discussion. This research was supported by the National Science Foundation under Grants No. NSF-PHY-1658693 and No. NSF-PHY-1806440 with additional engineering support from DOE Grant No. DE-SC0018229. J. W. F. and B. R. S. were supported in part by the DOE Early Career Grant No. DESC0019225, through computational resources and services provided by Advanced Research Computing at the University of Michigan, Ann Arbor, and by computational resources at the Lawrencium computational cluster provided by the IT Division at the Lawrence Berkeley National Laboratory, supported by the Director, Office of Science, and Office of Basic Energy Sciences, of the U.S. Department of Energy under Contract No. DE-AC02-05CH11231. Y. K. is supported in part by U.S. Department of Energy Grant No. DESC0015655. R. N. is supported by the National Science Foundation Graduate Fellowship under Grant No. DGE1746047. N. L. R. is supported by the Miller Institute for Basic Research in Science at the University of California, 
Berkeley. C.P.S. is supported in part by the National Science Foundation Graduate Research Fellowship under Grant No. 1122374. R. H. and K. A. R. are supported by the U.S. Department of Energy, Office of Science, Office of Nuclear Physics under Grants No. DEFG02-97ER41041 and No. DEFG02-97ER41033. We thank the University of North Carolina at Chapel Hill and the Research Computing group for providing computational resources and support that have contributed to these research results.

*These authors contributed equally to this work.

† salemi@mit.edu

fosterjw@umich.edu

§ouelletj@mit.edu

1winslow@mit.edu

[1] J. Preskill, M. B. Wise, and F. Wilczek, Cosmology of the invisible axion, Phys. Lett. 120B, 127 (1983).

[2] L. F. Abbott and P. Sikivie, A cosmological bound on the invisible axion, Phys. Lett. 120B, 133 (1983).

[3] M. Dine and W. Fischler, The not so harmless axion, Phys. Lett. 120B, 137 (1983).

[4] R. D. Peccei and H. R. Quinn, Constraints imposed by $C P$ conservation in the presence of instantons, Phys. Rev. D 16, 1791 (1977).

[5] R. D. Peccei and H. R. Quinn, $C P$ Conservation in the Presence of Instantons, Phys. Rev. Lett. 38, 1440 (1977).

[6] S. Weinberg, A New Light Boson?, Phys. Rev. Lett. 40, 223 (1978).

[7] F. Wilczek, Problem of Strong P and T Invariance in the Presence of Instantons, Phys. Rev. Lett. 40, 279 (1978).

[8] Sz. Borsanyi et al., Calculation of the axion mass based on high-temperature lattice quantum chromodynamics, Nature (London) 539, 69 (2016).

[9] P. Svrcek and E. Witten, Axions in string theory, J. High Energy Phys. 06 (2006) 051.

[10] L. Di Luzio, A. Ringwald, and C. Tamarit, Axion mass prediction from minimal grand unification, Phys. Rev. D 98, 095011 (2018).

[11] R. T. Co, F. D'Eramo, and L. J. Hall, Supersymmetric axion grand unified theories and their predictions, Phys. Rev. D 94, 075001 (2016).

[12] M. Tegmark, A. Aguirre, M. J. Rees, and F. Wilczek, Dimensionless constants, cosmology and other dark matters, Phys. Rev. D 73, 023505 (2006).

[13] J. I. Read, The local dark matter density, J. Phys. G 41, 063101 (2014).

[14] P. Sikivie, N. Sullivan, and D. B. Tanner, Proposal for Axion Dark Matter Detection Using an LC Circuit, Phys. Rev. Lett. 112, 131301 (2014).

[15] Y. Kahn, B. R. Safdi, and J. Thaler, Broadband and Resonant Approaches to Axion Dark Matter Detection, Phys. Rev. Lett. 117, 141801 (2016).

[16] P. Sikivie, Experimental Tests of the Invisible Axion, Phys. Rev. Lett. 51, 1415 (1983); Erratum, Phys. Rev. Lett. 52, 695 (1984).

[17] C. Hagmann, P. Sikivie, N. S. Sullivan, and D. B. Tanner, Results from a search for cosmic axions, Phys. Rev. D 42, 1297 (1990).
[18] S. Asztalos, E. Daw, H. Peng, L. J Rosenberg, C. Hagmann et al. (ADMX Collaboration), Large-scale microwave cavity search for dark-matter axions, Phys. Rev. D 64, 092003 (2001).

[19] N. Du, N. Force, R. Khatiwada, E. Lentz, R. Ottens et al. (ADMX Collaboration), Search for Invisible Axion Dark Matter with the Axion Dark Matter Experiment, Phys. Rev. Lett. 120, 151301 (2018).

[20] L. Zhong, S. Al Kenany, K. M. Backes, B. M. Brubaker, S. B. Cahn et al., Results from phase 1 of the HAYSTAC microwave cavity axion experiment, Phys. Rev. D 97, 092001 (2018).

[21] K. M. Backes et al. (HAYSTAC Collaboration), A quantumenhanced search for dark matter axions, Nature (London) 590, 238 (2021).

[22] D. F. Jackson Kimball et al., Overview of the cosmic axion spin precession experiment (CASPEr), Springer Proc. Phys. 245, 105 (2020).

[23] B. T. McAllister, G. Flower, E. N. Ivanov, M. Goryachev, J. Bourhill, and M.E. Tobar, The ORGAN experiment: An axion haloscope above $15 \mathrm{GHz}$, Phys. Dark Universe 18, 67 (2017).

[24] M. Silva-Feaver et al., Design overview of the DM radio pathfinder experiment, IEEE Trans. Appl. Supercond. 27, 1 (2017).

[25] A. Caldwell, G. Dvali, B. Majorovits, A. Millar, G. Raffelt, J. Redondo, O. Reimann, F. Simon, and F. Steffen (MADMAX Working Group), Dielectric Haloscopes: A New Way to Detect Axion Dark Matter, Phys. Rev. Lett. 118, 091801 (2017).

[26] A. V. Gramolin, D. Aybas, D. Johnson, J. Adam, and A. O. Sushkov, Search for axion-like dark matter with ferromagnets, Nat. Phys. 17, 79 (2021).

[27] S. Lee, S. Ahn, J. Choi, B. R. Ko, and Y. K. Semertzidis, Axion Dark Matter Search around 6.7 $\mu \mathrm{eV}$, Phys. Rev. Lett. 124, 101802 (2020).

[28] J. L. Ouellet et al., First Results from ABRACADABRA$10 \mathrm{~cm}$ : A Search for Sub- $\mu \mathrm{eV}$ Axion Dark Matter, Phys. Rev. Lett. 122, 121802 (2019).

[29] Superconducting Systems Inc., http://www .superconductingsystems.com.

[30] See Supplemental Material at http://link.aps.org/ supplemental/10.1103/PhysRevLett.127.081801 for more detail on the detector, calibrations, and analysis procedure.

[31] J.L. Ouellet et al., Design and implementation of the ABRACADABRA-10 cm axion dark matter search, Phys. Rev. D 99, 052012 (2019).

[32] J. W. Foster, N. L. Rodd, and B. R. Safdi, Revealing the dark matter halo with axion direct detection, Phys. Rev. D 97, 123006 (2018).

[33] COMSOL MULTIPHYSICS® v. 5.4. COMSOL AB, Stockholm, Sweden, http://www.comsol.com.

[34] Magnicon, http://www.magnicon.com/.

[35] J. Herzog-Arbeitman, M. Lisanti, P. Madau, and L. Necib, Empirical Determination of Dark Matter Velocities using Metal-Poor Stars, Phys. Rev. Lett. 120, 041102 (2018).

[36] M. Ackermann et al. (Fermi-LAT Collaboration), Search for gamma-ray spectral lines with the Fermi large area telescope 
and dark matter implications, Phys. Rev. D 88, 082002 (2013).

[37] A. Albert, G. A. Gomez-Vargas, M. Grefe, C. Munoz, C. Weniger, E. D. Bloom, E. Charles, M. N. Mazziotta, and A. Morselli (Fermi-LAT Collaboration), Search for $100 \mathrm{MeV}$ to $10 \mathrm{GeV} \gamma$-ray lines in the Fermi-LAT data and implications for gravitino dark matter in $\mu \nu \mathrm{SSM}, \mathrm{J}$. Cosmol. Astropart. Phys. 10 (2014) 023.

[38] M. Ackermann et al. (Fermi-LAT Collaboration), Updated search for spectral lines from galactic dark matter interactions with pass 8 data from the Fermi Large Area Telescope, Phys. Rev. D 91, 122002 (2015).

[39] V. Anastassopoulos et al. (CAST Collaboration), New CAST limit on the axion-photon interaction, Nat. Phys. 13, 584 (2017).

[40] C. Dessert, J. W. Foster, and B. R. Safdi, X-ray Searches for Axions from Super Star Clusters, Phys. Rev. Lett. 125, 261102 (2020).

[41] S. Chaudhuri, K. Irwin, P. W. Graham, and J. Mardon, Fundamental limits of electromagnetic axion and hidden-photon dark matter searches: Part I-The quantum limit, arXiv:1803.01627.
[42] Dark Matter New Initiatives FY2019, https://science .osti.gov/-/media/hep/pdf/Awards/Dark-Matter-NewInitiatives-FY-2019-List-of-Awards.pdf?la=en\&hash= 7134EDEA489651A3097DC8DB8C59069384A4538E.

[43] J.L. Ouellet et al., Probing the QCD axion with DMRadio-m ${ }^{3}$, Snowmass 2021 letter of interest, https://www .snowmass21.org/docs/files/summaries/CF/SNOWMASS21CF2_CF0-IF1_IF0_Ouellet-217.pdf.

[44] S. Chaudhuri et al., DMRadio-GUT: Probing GUT-scale QCD axion dark matter, Snowmass 2021 letter of interest, https://www.snowmass21.org/docs/files/summaries/CF/ SNOWMASS21-CF2_CF0-IF1_IF0_Saptarshi_Chaudhuri219.pdf.

[45] A.F. Leder et al., Magnet R\&D for low-mass axion searches, Snowmass 2021 letter of interest, https://www .snowmass21.org/docs/files/summaries/AF/SNOWMASS21AF5_AF0-CF2_CF0-IF1_IF0_Alexander_Leder-244.pdf.

[46] S. E. Kuenstner et al., Radio frequency quantum upconverters: Precision metrology for fundamental physics, Snowmass 2021 letter of interest, https://www.snowmass21.org/ docs/files/summaries/IF/SNOWMASS21-IF1_IF0-CF2_ CF0-193.pdf. 